\title{
Biological drugs targeting the immune response in the therapy of psoriasis
}

\author{
Saveria Pastore' \\ Emanuela Gubinelli \\ Luca Leoni \\ Desanka Raskovic ${ }^{2}$ \\ Liudmila Korkina' \\ 'Laboratory of Tissue Engineering \\ and Cutaneous Physiopathology; \\ ${ }^{2}$ Second Dermatology Unit, Istituto \\ Dermopatico dell'Immacolata, IRCCS, \\ Roma, Italy
}

\begin{abstract}
Chronic plaque psoriasis affects more than $2 \%$ of world population, has a chronic recurrent behavior, gives a heavy burden to the patients' quality of life, and hence remains a huge medical and social problem. The clinical results of conventional therapies of psoriasis are not satisfactory. According to the current knowledge of the molecular and cellular basis of psoriasis, it is defined as an immune-mediated chronic inflammatory and hyperproliferative skin disease. A new generation of biological drugs, targeting molecules and cells involved into perturbed pro-inflammatory immune response in the psoriatic skin and joints, has been recently designed and applied clinically. These biological agents are bioengineered proteins such as chimeric and humanized antibodies and fusion proteins. In particular, they comprise the antitumor necrosis factor- $\alpha$ agents etanercept, infliximab, and adalimumab, with clinical efficacy in both moderate-severe psoriasis and psoriatic arthritis, and the anti-CD11 a efalizumab with selective therapeutic action exclusively in the skin. Here, we overview recent findings on the molecular pathways relevant to the inflammatory response in psoriasis and present our clinical experience with the drugs currently employed in the dermatologic manifestations, namely etanercept, infliximab, and efalizumab. The growing body of clinical data on the efficacy and safety of antipsoriasis biological drugs is reviewed as well. Particular focus is given to long-term safety concerns and feasibility of combined therapeutic protocols to ameliorate clinical results.
\end{abstract}

Keywords: psoriasis, immune-mediated inflammation, etanercept, infliximab, efalizumab

\section{Introduction}

Psoriasis is one of the most common chronic and recurrent dermatoses affecting approximately $2 \%$ of the general population in Western countries. The incidence of psoriasis does not depend on the sex. The disease can affect people of all ages, although its onset can occur in early adulthood. Approximately $10 \%-30 \%$ of patients with psoriasis develop psoriatic arthritis (PsA). Pretty often, psoriasis is associated with systemic disorders such as Crohn's disease, type 2 diabetes, and metabolic syndrome. The association of psoriasis and cardiovascular pathologies represents an emerging concern for patients with moderate-severe psoriasis (Griffiths and Barker 2007). Clinically, psoriasis vulgaris is characterized by well-demarcated erythema and scaly skin plaques. The psoriatic lesions often occur at sites of epidermal trauma, such as elbows and knees, and are often symmetrical. Morphological features of chronic plaque psoriasis include marked increase in keratinocyte proliferation, abnormal pattern of keratinocyte differentiation, significant alterations in dermal capillary vasculature, and prominent inflammatory cell infiltration of both the dermis and epidermis. The etiology and pathogenesis of psoriasis remain unclear so far. It is thought that psoriasis and associated PsA belong to complex pathologies with both inherited and acquired components. There is evidence of familial traits and association of psoriasis with human leukocyte antigen (HLA) antigens (Travers 2000). Thorough genome-wide genetic analyses of affected families have revealed some susceptibility loci for psoriasis and PsA (Valdimarsson 2007). However, corresponding genes still have to be identified. 
Much attention is currently paid to the characterization of the single susceptibility genes in order to early diagnose and predict severity of this disorder, improve its treatment, and eventually prevent its onset (Liu et al 2007). The acquired character of psoriasis is confirmed by numerous observations that its onset is often associated with trauma, stress, bacterial and viral infections, administration of proinflammatory drugs such as interferons (IFNs), or with rapid withdrawal of immunosuppressive drugs, for example, corticosteroids (Griffiths and Barker 2007). It is generally assumed that unbalanced immune responses are major players in the disease process. The perturbed immune responses seem to contribute to the two major features of the psoriatic skin, persistent inflammation and hyperproliferation of keratinocytes (Bruch-Gerharz et al 2003). The exact sequence of events, as well as the molecular mediators that lead to inflammatory and hyperproliferative responses are yet to be defined. However, both systemic and topical preparations with clinical efficacy in psoriasis possess immuno-modulating properties with both antiinflammatory and antiproliferative action.

In the present paper, we will overview the data on the pathogenesis of chronic immune-mediated inflammation in the psoriatic skin, with special emphasis on the molecular targets for the recently approved and clinically effective biological drugs. The Italian experience of clinical application of biological drugs in the patients with moderate/severe forms of psoriasis will also be described.

\section{Pathogenesis of chronic inflammation in the psoriatic skin}

The persistence of the psoriatic lesion is currently viewed as depending on a vicious proinflammatory circuit interactively sustained both by resident cell populations of the skin and infiltrating leukocytes. The latter relies on the relentless immune cell-driven expression of a complex system of proinflammatory cytokines and potent chemoattractants by epidermal keratinocytes. This circuit is currently assumed as the basic paradigm of $\mathrm{T}$ cell-based skin inflammation, and has been well documented in the active lesion of psoriasis (Pastore et al 2006). However, until recently, no specific causes for the initiation of this disease were recognized. At a cellular level, the accumulation of inflammatory cells largely consisting of activated T cells and plasmacytoid dendritic cells precedes other microscopic and clinical symptoms of the pathology. This observation led to the hypothesis of the autoimmune character of psoriasis (Baumgarth and Bevins 2007). According to this study, the antimicrobial peptide cathelicidin, LL37, which is strongly represented in psoriatic lesions (Ong et al 2002), binds to the DNA leaked from damaged cells in the lesional epidermis and forms an immunogenic complex that breaks tolerance and induces the production of IFN- $\alpha$ by plasmacytoid dendritic cells (Lande et al 2007). The molecular pathways underlying peculiar susceptibility of the psoriatic skin to trigger this reaction, including the early and preferential recruitment of plasmacytoid dendritic cells, remain elusive so far.

\section{Central role of TNF- $\alpha$ in psoriatic inflammation}

Tumor necrosis factor- $\alpha$ (TNF- $\alpha)$ is crucially involved in the pathogenesis of many human diseases, including sepsis, cancer and inflammatory disorders of the gut, joint, and skin. The central role of this cytokine in psoriasis has definitely come to light through the observations of the efficacy of anti-TNF- $\alpha$ biological therapies (Girolomoni et al 2002). Virtually all cell types can be induced to express TNF- $\alpha$, although activated dendritic cells, monocytes, macrophages, neutrophils, and $\mathrm{T}$ cells are by far its richest sources. Notably, both IFN- $\gamma$-secreting type-1 helper T (Th1) cells and interleukin (IL)-4 type-2 helper T- (Th2) cells release relevant levels of this potent pro-inflammatory cytokine, indicating its participation to all $\mathrm{T}$ cell-mediated reactions. For all T cell subpopulations, TNF- $\alpha$ acts as a major inducer of functional maturation. The sequence of events leading to the TNF- $\alpha$-driven inflammatory response in the skin is schematized in Figure 1. In the early phases of skin response to physical and chemical insults, perivascular mast cells degranulate, releasing a plethora of presynthesized mediators that includes TNF- $\alpha$ (Figure 1A). Mast cell-produced mediators trigger fast activation of the endothelial cells, which consequently up-regulate adhesion molecules and cytokines (Figure 1B). This up-regulation favors leukocyte adherence to vascular endothelium, rolling, and extravasation (Figure 1C). The activated leukocytes recruited into the skin release huge amount of TNF- $\alpha$. Epidermal keratinocytes themselves are modest producers of TNF- $\alpha$, but they respond to this cytokine with an articulate program of de novo expression and release of other pro-inflammatory cytokines, chemokines, and growth factors (Figure 1D) (Pastore et al 2006). The growth factors include vascular endothelial growth factors and the angiopoietins responsible of the characteristic abnormal dermal vascular proliferation and angiogenesis (Griffiths and Barker 2007).

Bioactive TNF- $\alpha$ can be found in two forms, a membranebound form and a proteolytically solubilized form. Its biological effects are mediated by two cell surface receptors, 


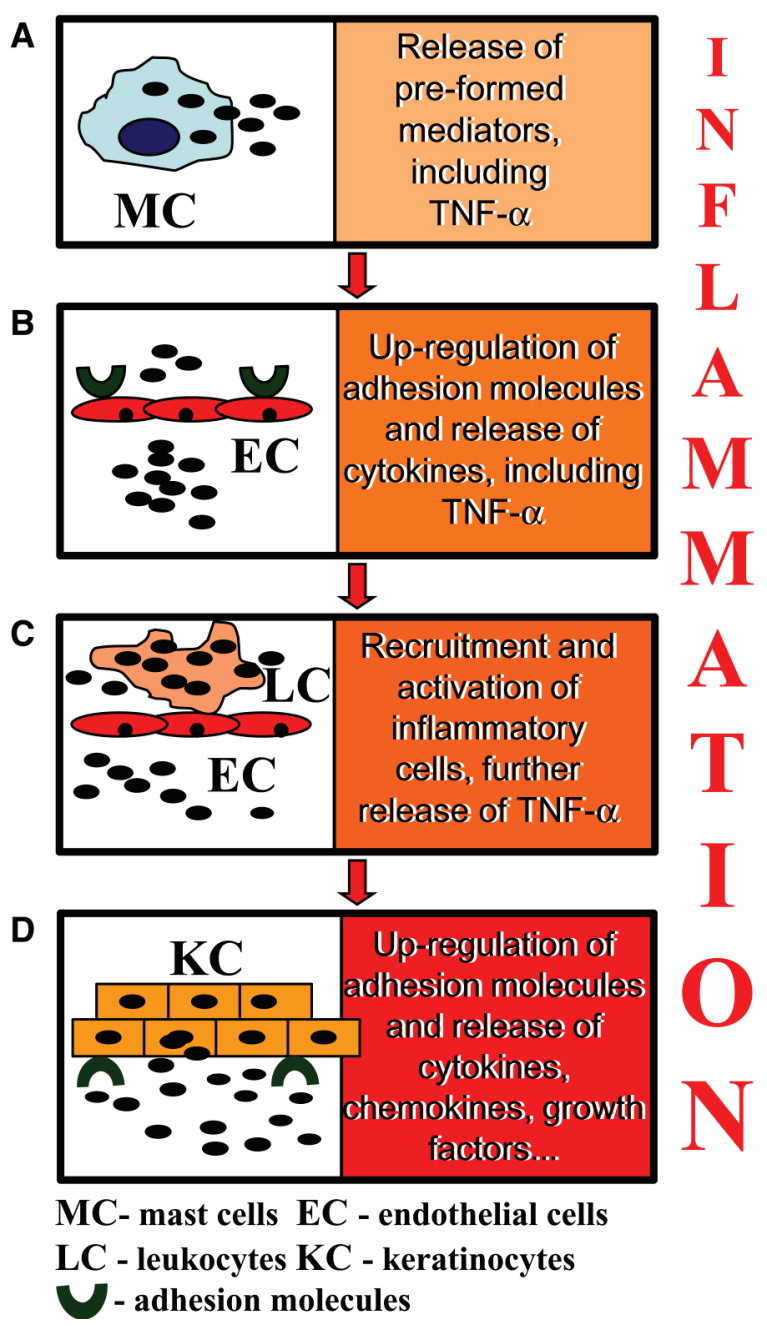

Figure I TNF- $\alpha$-driven inflammatory cascade in the skin. Among the pre-formed mediators released by mast cells (A),TNF- $\alpha$ boosts the pro-inflammatory activation of resident cell populations which include endothelial cells (B). In their turn, endothelial cells respond to TNF- $\alpha$ with up-regulated expression of surface adhesion molecules, which facilitate the adhesion and migration of leukocytes to peripheral tissues, and a new wave of leukocyte-derived cytokine release (C). Eventually, skin keratinocytes amplify the inflammatory response at the local level, with massive release of cytokines, chemokines, and growth factors (D).

Abbreviations: TNF- $\alpha$, tumor necrosis factor- $\alpha$.

respectively. One is the ubiquitously expressed TNF-R1 (synonyms, p55 and CD120a) and the other one is TNF-R2 known also as $\mathrm{p} 75$ or CD120b. The role of TNF-R1 in skin inflammation has been confirmed experimentally (Pasparakis et al 2002). TNF-R2 is predominantly found on hematopoietic and endothelial cells and crucially implicated in the TNF$\alpha$-driven cell apoptosis (Locksley et al 2001). Both soluble and membrane-bound forms of TNF- $\alpha$ are the molecular targets for biological therapy of psoriasis. In particular, the antiTNF- $\alpha$ drugs used for the therapy of psoriasis are based either on anti-TNF- $\alpha$ monoclonal antibodies such as infliximab or on TNF-R-based reagents such as etanercept (Gisondi et al 2004) (Figure 2A). They were first used for the treatment of patients with moderate-to-severe rheumatoid arthritis who failed to respond to conventional therapies. Infliximab is a chimeric anti-TNF- $\alpha$ monoclonal immunoglobulin G1a (IgG1a) antibody, with a human constant region and a murine variable portion. Infliximab binds and neutralizes the soluble form of TNF- $\alpha$ with extremely high affinity. It also binds the membrane-bound form, with lower affinity though. Thus, by binding to TNF- $\alpha$, infliximab triggers the elimination of TNF- $\alpha$-producing cells by both complement-mediated and antibody-dependent, cytotoxic mechanisms. Infliximab is also currently approved by the United States Food and Drug Administration (US FDA) to be used in combination with methotrexate for the treatment of active rheumatoid arthritis and Crohn's disease. Etanercept is a genetically engineered fusion protein consisting of an homodimer of the extracellular portion of TNF-R p 75 subunit fused with the constant region of human IgG1. The complexes of TNF- $\alpha$ with etanercept are quite unstable. Nonetheless, they diminish or even prevent the biological actions of the soluble forms of TNF- $\alpha$. Etanercept is the US FDA-approved drug for the treatment of psoriatic arthritis, rheumatoid arthritis, and polyarticularcourse juvenile rheumatoid arthritis. Finally, the genetically engineered recombinant human $\operatorname{IgG1}$ monoclonal antibody adalimumab, which binds both soluble and membrane-bound TNF- $\alpha$ with high affinity, is presently limited to the therapy of active, progressive psoriatic arthritis that failed to respond to one or more antirheumatic drugs.

\section{Psoriasis as a T cell-mediated skin disorder}

Cytokines of the Th1 pathway, including IFN- $\gamma$, IL-2, and IL-12 are abundantly expressed in the psoriatic plaques. Therefore, psoriasis is typically classified as a Th1-driven disease. The central role of $\mathrm{T}$ cells has been proven many years ago because cyclosporin $\mathrm{A}$, a $\mathrm{T}$ cell-targeted immune suppressive drug, had been found clinically effective in the treatment of psoriasis (Mueller and Herrmann 1979). Further evidence has come from the transmission of psoriasis by bone marrow transplantation (Wahie et al 2006). As a first step, T cells undergo extravasation and subsequent migration into the lesion, being recruited by chemotactic and adhesion molecule-mediated signals (Schon and Ludwig 2005). Then, their functional activation in the affected skin takes place, which leads to massive release of proinflammatory mediators and activation of cytotoxic mechanisms. Thus, further T cell-mediated damage to the skin occurs. Expression of integrin LFA-1 (the leukocyte function-associated antigen) on $\mathrm{T}$ cell membrane appears to play a central role 


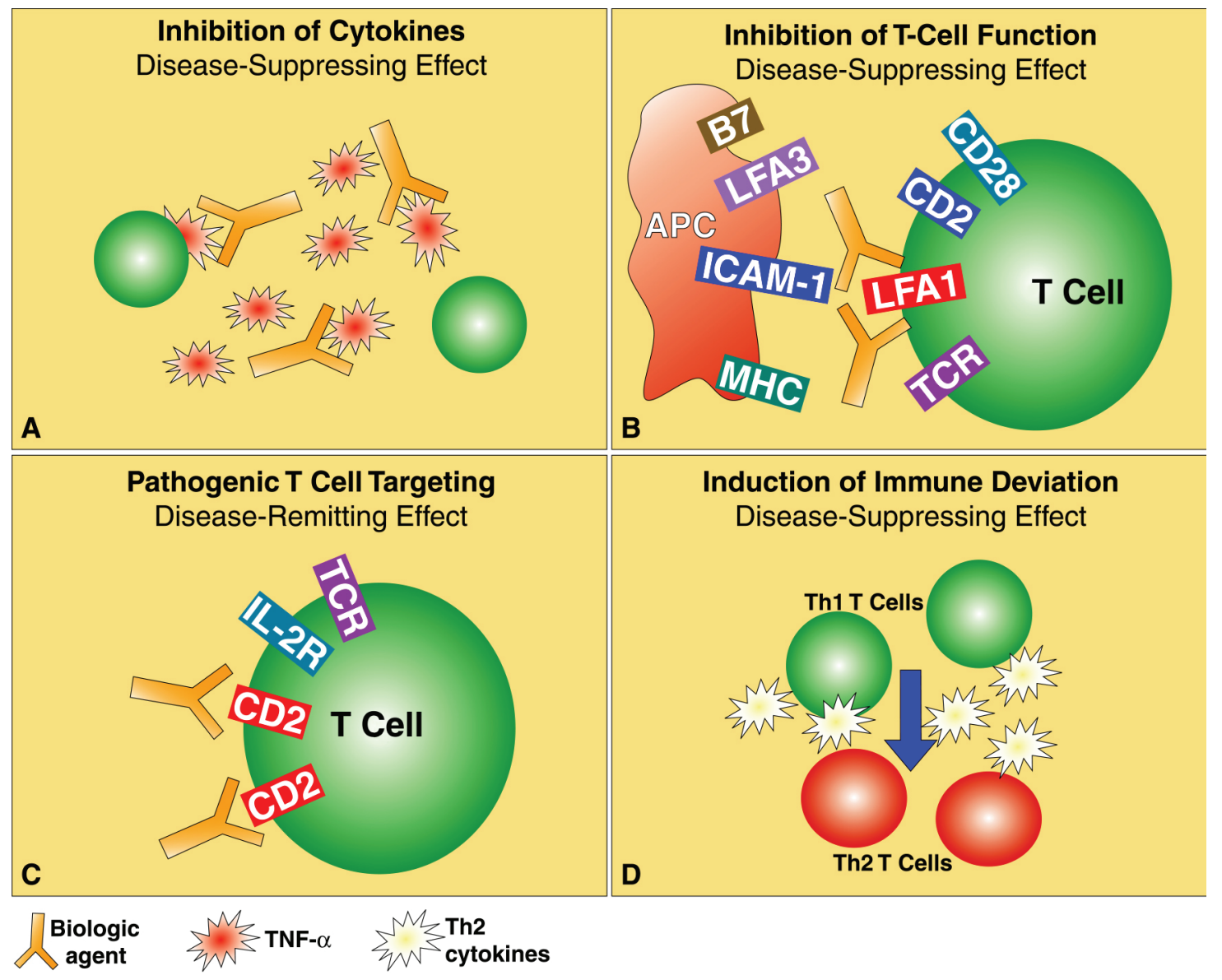

Figure 2 Strategies for targeted biological therapy of psoriasis. These include existing and potential biological drugs for the therapy of psoriasis, such as anti-TNF- $\alpha$ (A) or anti-LFA-I (B) antibodies, inhibitors of CD2 expression on the surface of activated pathogenic T cells (C), or cytokines to balance the Th I-skewed immune response (D), these last presently under investigation.

Abbreviations: APC, antigen-presenting cell; LFA-I, lymphocyte function-associated antigen- I; TNF- $\alpha$, tumor necrosis factor- $\alpha$.

for both $\mathrm{T}$ cell extravasation and functional activation. The LFA-1 integrin binds to the cognate ligand, the intercellular adhesion molecule (ICAM)-1, expressed on the surface of the endothelial and epithelial cells as well as on antigenpresenting cells. This binding maximally activates $\mathrm{T}$ cell functions (Hogg et al 2003), and it is specifically targeted by efalizumab, the humanized monoclonal antibody that binds to the CD1 1 a portion of LFA-1 currently used as a relatively safe and effective biological drug for the therapy of psoriasis (Figure 2B). Another molecular interaction implicated in the functional activation of $\mathrm{T}$ cells is the binding of the costimulatory receptor CD2 on $\mathrm{T}$ cell surface, to its ligand LFA-3 on antigen-presenting cells. Indeed, the first biological drug approved by the US FDA for the therapy of chronic plaque psoriasis was alefacept, a fusion protein formed by the binding site of LFA-3 and the constant portion of the human IgG, that hence targets and neutralizes the function of $\mathrm{CD} 2$ (Figure 2C). Finally, the possibility to balance the Th1 deviation of the immune response with the administration of
Th2-skewing cytokines is currently under investigation for the therapy of psoriasis (Figure 2D).

\section{Clinical results of the use of biological drugs in the therapy of psoriasis}

The biological drugs currently available in Europe with the explicit indication to treat psoriasis are etanercept, infliximab, and efalizumab. The clinical response to therapy in psoriatic patients is expressed as a percent of improvement in the Psoriasis Area and Severity Index (PASI) score. Other quantitative standard parameters employed to describe general results of the biological therapies are the Dermatology Life Quality Index (DLQI), Physician's Global Assessment (PGA), and the Health-Related Quality-Of-Life (HRQOL) (Shikiar et al 2006). Currently, biological therapies are applicable to psoriatic patients with a PASI score $\geq 10$, a DLQI score $\geq 10$, and a clinical history of lack of response, intolerance, or contraindication to standard systemic therapies. 
In the paragraphs below, we will overview data on the clinical efficacy, briefly summarized in Table 1, of these three biologics administered to the patients with severe-tomoderate forms of plaque psoriasis. Their safety profiles can be compared in Table 2. The effects of biological drugs on the quality of life of psoriatic patients will be discussed in detail. While these biological agents may possess greater efficacy in treating moderate-to-severe psoriasis, there is a higher cost associated with their administration compared to phototherapy and traditional systemic treatments (Hankin et al 2005), with important cost differences for different biological drugs (Nelson et al 2008). Furthermore, because of the chronic nature of psoriasis, these costs accumulate. A deeper analysis showed (Nelson et al 2008) that currently recommended treatment regimens for these medications are far from being optimal with regard to their cost/efficacy ratio. The implementation of economic analysis in the phase of determining the treatment regimen seems to be essential.

\section{Etanercept}

Etanercept is a soluble TNF- $\alpha$ antagonist that competitively inhibits the interaction of TNF- $\alpha$ with cell-surface receptors. The impaired interaction with receptors prevents TNF- $\alpha$-mediated cellular responses and modulates the activity of other TNF- $\alpha$-regulated pro-inflammatory cytokines. In agreement with the Guidelines of the British Association of Dermatologists (BAD), Etanercept is recommended as the anti-TNF agent of choice for patients with moderate-to-severe stable psoriasis (Smith et al 2005). The drug (etanercept, Enbrel ${ }^{\circledR}$ ) was approved by the US FDA for the treatment of patients with psoriasis, PsA, rheumatoid arthritis (RA), juvenile idiopathic arthritis, and ankylosing spondylitis. With regard to psoriasis, the European Agency for the Evaluation of Medicinal Products (EMEA) has approved etanercept for the treatment of adults with moderate-to-severe psoriasis who failed to respond to, had a contraindication to, or were intolerant to other systemic therapy, including cyclosporin, methotrexate, or psoralen and ultraviolet A (PUVA).

More than an eight years long clinical experience with etanercept in the treatment of RA has demonstrated its high therapeutic potential, safety, and patients' compliance (Krueger et al 2006). The therapy with etanercept results in a substantial improvement in pruritus and health-related quality of life parameters. The recommended starting doses are injections of $25 \mathrm{mg}$ or $50 \mathrm{mg}$ of etanercept twice a week (BIW) for the first 12 weeks, followed, if necessary, by $25 \mathrm{mg}$ BIW. The total duration of etanercept course depends on the clinical response of the patient but should not be longer than 24 weeks. The phase II (Gottlieb et al 2003a) and phase III (Leonardi et al 2003; Papp et al 2005) clinical trials, which had enrolled more than 1000 patients with moderate-to-severe chronic plaque psoriasis, have demonstrated that high starting dose of etanercept ( $50 \mathrm{mg}$ BIW) resulted in the faster onset of therapeutic action and the higher efficacy rate as compared to low starting dose (25 mg BIW). After 12 weeks of treatment, $34 \%$ of patients treated with $25 \mathrm{mg}$ etanercept BIW, achieved more than 75\% improvement in PASI (PASI-75) compared with $49 \%$ of those receiving $50 \mathrm{mg}$ BIW. At the cessation of the trials (24 weeks), 44\% of the patients receiving $25 \mathrm{mg}$ etanercept BIW and $59 \%$ of the patients who started with $50 \mathrm{mg}$ etanercept BIW achieved or maintained a PASI-75 response, respectively (Leonardi et al 2003; Papp et al 2005). These data suggested that the initial dose of $50 \mathrm{mg}$ etanercept BIW is the optimal therapeutic approach in psoriasis with regard to clinical parameters and disease severity (Boehncke et al 2006), with a tapering of the therapeutic dosage to $25 \mathrm{mg}$ BIW after 12 weeks for patients achieving a PASI-75

Table I Short-term efficacy of biological drugs in the therapy of psoriasis

\begin{tabular}{|c|c|c|c|c|}
\hline Agent & Study & Dosing & $\begin{array}{l}\text { Time to primary } \\
\text { end point }\end{array}$ & $\begin{array}{l}\text { Results (\% of patients } \\
\text { reaching PASI75) }\end{array}$ \\
\hline Etanercept & Gottlieb et al 2003a & $2 \times 25 \mathrm{mg}$ weekly SC & Week 12 & $30 \%$ \\
\hline Etanercept & Leonardi et al 2003 & $\begin{array}{l}\mathrm{I} \times 25 \mathrm{mg} \text { vs } 2 \times 25 \mathrm{mg} \text { vs } \\
2 \times 50 \mathrm{mg} \text { weekly SC }\end{array}$ & Week 12 & I $4 \%$ vs $34 \%$ vs $49 \%$ \\
\hline Infliximab & Chaudhari et al 200I & $\begin{array}{l}5 \mathrm{mg} / \mathrm{kg} \text { vs } \\
10 \mathrm{mg} / \mathrm{kg} \text { IV at weeks } 0,2 \text { and } 6\end{array}$ & Week 10 & $82 \%$ vs $73 \%$ \\
\hline Infliximab & Gottlieb et al 2004 & $\begin{array}{l}3 \mathrm{mg} / \mathrm{kg} \text { vs } \\
5 \mathrm{mg} / \mathrm{kg} \mathrm{IV} \text { at weeks } 0,2 \text { and } 6\end{array}$ & Week 10 & $72 \%$ vs $88 \%$ \\
\hline Efalizumab & Gordon et al 2003 & I mg/kg weekly SC & Week 12 & $27 \%$ \\
\hline Efalizumab & Lebwohl et al 2003 & $\mathrm{I} \mathrm{mg} / \mathrm{kg}$ vs $2 \mathrm{mg} / \mathrm{kg}$ weekly SC & Week I2 & $22 \%$ vs $28 \%$ \\
\hline Efalizumab & Menter et al 2005 & I mg/kg weekly SC & Week 12 & $27 \%$ \\
\hline
\end{tabular}


Table 2 Safety profiles of the biological agents in the therapy of psoriasis

\begin{tabular}{lll}
\hline Agent & Common side events & Potential serious adverse events \\
\hline Etanercept & I. Injection site reaction & I. Serious infections \\
2. Respiratory tract infection & 2. Pancytopenia and aplastic anemia \\
3. Headache & 3. Demyelinating disorders \\
4. Nausea & 4. Congestive heart failure or its worsening \\
5. Dizziness & 5. Auto-antibody formation and lupus-like syndrome \\
6. Cough & 6. Possible increased risk of malignancy \\
7. Abdominal pain & \\
8. Rash & \\
I. Respiratory tract infections & \\
2. Nausea & I. Infusion reaction \\
3. Abdominal pain & 2. Serious infections, including reactivation of latent tuberculosis \\
4. Dyspepsia & 3. Demyelinating disorders \\
5. Fever & 4. Congestive heart failure or its worsening \\
6. Fatigue & 5. Autoantibody formation and lupus-like syndrome \\
7. Headache & 6. Possible increased risk of malignancy \\
8. Rash & \\
I. First dose reaction complex & \\
2. Headache & I. Thrombocytopenia \\
3. Chills & 2. Psoriasis worsening \\
4. Fever & 3. Rebound psoriasis \\
5. Nausea & 4. Serious infections \\
6. Myalgia & 5. Possible increased risk of malignancy \\
& 6. Inflammatory arthritis \\
& 7. Hypersensitivity reaction \\
\hline
\end{tabular}

response. A starting dosage of $100 \mathrm{mg}$ of etanercept once a week (OW) for 12 weeks has been evaluated in a limited clinical study (Cassano et al 2006). In this comparative pilot study, 108 patients with plaque psoriasis were enrolled and allocated into two experimental groups, one treated with $50 \mathrm{mg}$ BIW and one with $100 \mathrm{mg}$ OW. After 12 weeks, the efficacy and tolerability parameters were compared. The authors have drawn conclusions that the two dosage regimens were similar.

After the 24 week-long course of etanercept therapy, the time of the disease relapse ranges from 70 to 91 days and appears to be dose-related (Smith et al 2006). In case of relapse, the therapy with etanercept can be repeated without loss of drug efficacy (Gottlieb et al 2003a). Etanercept is generally well tolerated and the compliance of the patients is quite high. Although laboratory monitoring is not mandatory for patients treated with etanercept, thorough laboratory tests are strongly recommended before starting the therapy to exclude re-activation of tuberculosis, human immunodeficiency virus, and hepatitis. There are several reports documenting that etanercept may be a safe option for treating patients suffering from hepatitis C (Magliocco and Gottlieb 2004; Rokhsar et al 2006). An accurate patient history is generally sufficient to recognize other standard limitations for the therapy with etanercept, such as demyelinating neurological disorders, malignancy and severe congestive heart failure (Boehncke et al 2006). In the everyday clinical practice, the most common immediate adverse event is the reaction at the site of injection, particularly during the first month of therapy. Usually, this event tends to resolve without any medical intervention. In general, etanercept as all the other biologics should be prescribed by a dermatologist with vast experience in the management of difficult-to-treat and resistant forms of psoriasis. In our personal experience, we carry out an accurate screening program before starting the therapeutic course with biological drugs. Thorough monitoring is also performed every 6-8 weeks during the treatment period and every 12 weeks in the follow-up period.

\section{Infliximab}

In November 2005, Centocor Inc. announced that the US FDA accepted filing of a supplemental Biologics License Application (sBLA) for the use of infliximab in the treatment of moderate-to-severe plaque psoriasis. The acceptance of this document followed the September 2005 European Commission approval of infliximab for the treatment of moderate-to-severe plaque psoriasis in adults who failed to respond to, had a contraindication to, or were intolerant of other systemic therapy including cyclosporin A, methotrexate, or PUVA. The drug has been shown effective also in the treatment of erythrodermic and pustular psoriasis (Lewis et al 2005). 
This biological drug should be prescribed as a monotherapy and administered intravenously (iv) at doses of 3,5 , or $10 \mathrm{mg} / \mathrm{kg}$ three times, at week 0,2 , and 6 . This protocol results in a rapid and significant clearance of psoriatic lesions as compared to the placebo. At week 10, after the three-dose induction regimen, $82 \%$ and $73 \%$ of patients treated with $5 \mathrm{mg} / \mathrm{kg}$ and $10 \mathrm{mg} / \mathrm{kg}$, respectively, achieved PASI-75. The same PASI-75 was observed in $18 \%$ of patients in the placebo group. These data were obtained in a small phase II, randomized, double-blind clinical trial (Chaudhari et al 2001). No significant differences in the clearing of disease were observed between doses of 5 and $10 \mathrm{mg} / \mathrm{kg}$ as induction regimen, although the dose of $10 \mathrm{mg} / \mathrm{kg}$ led to a longer remission (Gottlieb et al 2003b). In another phase II, randomized, double-blind study, the maximum response to infliximab was observed at week 10 . The percentage of patients achieving PASI-75 at this time-point was $72 \%, 88 \%$, and $6 \%$ for the groups treated with $3 \mathrm{mg} / \mathrm{kg}, 5 \mathrm{mg} / \mathrm{kg}$ and placebo, respectively. This highly positive clinical result was maintained until week 14 after discontinuation of therapy in the $5 \mathrm{mg} / \mathrm{kg}$ group. In the group treated with $3 \mathrm{mg} / \mathrm{kg}$, relapses occurred after 10 weeks, suggesting that a maintenance dose every 8 weeks should be considered after the initial induction regimen (Gottlieb et al 2004). In the extended multicenter European Infliximab for Psoriasis Efficacy and Safety Study (EXPRESS), $61 \%$ and $45 \%$ of patients receiving initial doses of $5 \mathrm{mg} / \mathrm{kg}$ at weeks 0,2 and 6 and, then, every 8 weeks, achieved PASI-75 and PASI-90, respectively, at week 50. These data confirmed the efficacy of maintenance regimens over 1 year in the treatment of moderate-to-severe psoriasis (Reich et al 2005). Recently, in order to define the lowest effective infliximab dose and the largest interval between two consecutive administrations, two maintenance protocols (continuous and intermittent) were compared (Menter et al 2007). Patients were randomized for the induction therapy with $3 \mathrm{mg} / \mathrm{kg}, 5 \mathrm{mg} / \mathrm{kg}$ infliximab or placebo. All infliximabtreated participants were further randomized at week 14 for the continuous or intermittent maintenance regimens with the same doses used during the induction period. At week 10, $70.3 \%$ and $37.1 \%$ percent of patients treated with $3 \mathrm{mg} / \mathrm{kg}$, and $75.5 \%$ and $45.2 \%$ of patients receiving $5 \mathrm{mg} / \mathrm{kg}$, achieved PASI-75 and PASI-90, respectively, versus 1.9\% (PASI-75) and $0.5 \%$ (PASI-90) of patients receiving the placebo. At week 50, the PASI responses were better maintained with continuous bimonthly therapy than with intermittent, "at request" maintenance protocol. The best PASI values were achieved in the group treated with $5 \mathrm{mg} / \mathrm{kg}$ infliximab. The safety profile in this study was similar to that observed in the previous trials. During the induction period, a higher incidence of adverse events and infusion reactions in both the infliximab-treated groups was observed as compared to the placebo group. However, an increased incidence of infusion reactions in the $3-\mathrm{mg} / \mathrm{kg}$ infliximab administered "at request" was observed. Two cases of tuberculosis and 2 cases of lupus-like syndrome were observed in patients treated with infliximab, whereas lymphoproliferative disorders or demyelinating conditions were not reported.

Different adverse events were observed in clinical trials with infliximab. The most common were infusion-related immediate reactions occurring during, or within 1 hour after, intravenous infusion. These included urticaria, fever, hypo/hypertension, and very rarely anaphylaxis. Twenty percent of patients treated with infliximab developed an infusion reaction compared with $10 \%$ of patients receiving the placebo. Thus, a need of antihistamine and antipyretic pretreatment before infusion was justified. Patients positive for antiinfliximab antibodies were more likely to have an infusion reaction as compared to those who were negative (Menter et al 2005). Like all TNF- $\alpha$ inhibitors, infliximab is an immunosuppressive agent and hence is associated with the occurrence of infections, lupus-like syndrome, demyelinating conditions, and a possible increase in lymphoma risk. The infections, particularly the reactivation of latent tuberculosis and other opportunistic infections, are a major concern in patients treated with this biological drug. Indeed, clinical trials showed that $36 \%$ of patients receiving infliximab developed infections versus $25 \%$ of patients in the placebo group (Weinberg 2006). These data confirm the necessity for rigorous screening of the infection before treatment, including a PPD skin test and chest radiographs. In the follow-up period, symptoms like persistent fever, weight loss and night sweats should be taken into consideration. A prophylaxis of latent infections should be done routinely. A meta-analysis of rare harmful adverse effects observed in randomized trials on the safety of anti-TNF- $\alpha$ therapy has demonstrated an increased risk of infections and a dose-dependent risk of blood cell, skin, and solid organ tumours (Bongartz et al 2006). In contrast, the results from the British Society for Rheumatology Biologics Register showed that in clinical practice, anti-TNF- $\alpha$ therapy did not increase the risk of malignancy in the low risk patients (Dziadzio and Smith 2007). Several cases of exacerbation of demyelinating diseases and heart failure have also been reported. Therefore, the patients having an anamnesis of either central or peripheral demyelinating disorders and/or congestive heart failure usually are excluded from the treatment with anti-TNF- $\alpha$ drugs 
(Hochberg et at 2005). As adverse effects of anti-TNF- $\alpha$ therapy in psoriasis, liver toxicity, or cholestasis have been reported. However, some uncertainties regarding these associations remained, because psoriatic patients could be predisposed to transaminase abnormalities and the majority of them were concomitantly treated with hepatotoxic drugs. Haematologic disorders such as leukopenia, neutropenia, trombocytopenia and pancytopenia could be associated with Infliximab administration (Zeichner and Lebwohl 2007).

To date, neither increased risk of embryotoxicity nor teratogenicity, nor adverse pregnancy outcome (such as birth defects, premature birth, and low birth weight) have been reported in arthropatic patients treated with anti-TNF therapy versus the general population (Skomsvoll et al 2007). However, the available data are limited. Until more data are available, no firm conclusions can be drawn regarding the safety of anti-TNF therapy in pregnancy. Anti-TNF agents are not usually used during lactation, although the risk of toxicity is probably negligible (Skomsvoll et al 2007).

\section{Efalizumab}

The efficacy and safety of efalizumab in the treatment of psoriasis has been evaluated in numerous studies. The patients treated, all adults with moderate to severe plaque psoriasis and candidates for systemic therapy, experienced a greater improvement in PASI-75 and PASI-50 than the placebotreated group. The best clinical results were obtained with weekly $1 \mathrm{mg} / \mathrm{ml}$ subcutaneous injections of efalizumab. After 12 weeks of therapy, $59 \%$ and $27 \%$ of patients achieved PASI-50 and PASI-75, respectively. In the placebo group, the values of PASI-50 and PASI-75 were observed in the $14 \%$ and $4 \%$ of the patients, respectively. The efalizumab-treated group of patients also showed a greater improvement from the baseline in the DLQI overall score with respect to the placebo group (47\% versus 14\%). The therapy was well tolerated with neither evidence of systemic toxicity, nor increases in infection or malignancy (Gordon et al 2003). Another phase III randomized double-blind placebo-controlled study has shown similar efficacy and safety profiles when the treatment with efalizumab was extended from 12 to 24 weeks (Lebwohl et al 2003). The long-term continuous efalizumab therapy not only sustained the efficacy without increasing cumulative and organ toxicity, but also resulted in additional clinical improvement, as demonstrated in two other clinical trials. In-fact in a phase III randomized double-blind placebocontrolled trial followed by an open-label study, 26.6\% and $58.5 \%$ of patients, achieving PASI-75 and PASI-50 after 12 weeks therapy with the $1 \mathrm{mg} / \mathrm{kg}$ dose, rose to $43 \%$ and
$66 \%$ after 24 weeks of continued treatment, respectively (Menter et al 2005). In a consecutive open-label trial, the PASI improvement was maintained throughout the 30-month treatment period (Gottlieb et al 2006).

The median time between discontinuation of efalizumab and aggravation of psoriasis was 58 days. The efficacy and safety of a second treatment with efalizumab in patients achieving PASI-75 at week 12 showed a mean PASI improvement of $62.3 \%$ from the study baseline (Sterry et al 2006). Efalizumab was shown to provide a similar significant clinical improvement in patients where at least one systemic therapy had failed or was contraindicated (Dubertret et al 2006). Efalizumab was generally well tolerated, with similar incidence and types of adverse events in the treated and placebo groups. The most frequent and short-term adverse event reported in all these clinical studies was a flu-like syndrome with fever, nausea, chills, and headache, commonly accompanying the first dose and disappearing by the third dose of drug administration. Regarding the incidence of infections, no significant difference between the efalizumabtreated group (29\%) and the placebo-group (26\%) was shown, and the frequency of infections did not increase with the extended therapy up to 27 months. Thorough analysis of the clinical trials with efalizumab did not reveal cases of re-activation of latent tuberculosis, Pneumocystis carinii, histoplasmosis, or toxoplasmosis infections (Langley et al 2005). Although thrombocytopenia ( $0.3 \%$ of patients treated) and hemolytic anemia represented rare adverse events, the monitoring of platelet count is recommended. Efalizumab does not appear to increase the risk of arthropathy, as was shown in the long-term studies up to three years of continuous treatment (Pincelli et al 2006). Up to now, there are no reports on an increased risk of malignancy in psoriasis patients treated with efalizumab. Nonetheless, caution is strongly recommended when any immune suppressive drug is used.

A rather peculiar exacerbation of psoriasis can occur during treatment with efalizumab. In a report by an English group (Menter and Griffiths 2007), up to 20\% of the patients developed mild, transient, papular flare localized on body folds. These skin lesions were easily managed with topical steroids. In addition, $5 \%$ of nonresponders to the therapy developed severe generalized inflammatory flare within 6-10 weeks, which required systemic therapy with methotrexate and/or discontinuation of the therapy with efalizumab. Thirteen per cent of the patients who stopped the efalizumab therapy abruptly developed a rebound syndrome, defined as a $>125 \%$ increase in severity compared with pretreatment baseline, or as a worsening 
of the form of psoriasis. It should be stressed that the majority of these patients failed to achieve a PASI-50 response after 12 weeks of efalizumab treatment (Papp 2006).

\section{The Italian experience with biological drugs}

In order to evaluate the long-term results of the current treatments of moderate-to-severe psoriasis, the research program PSOCARE was recently promoted by the Italian Drug Agency (AIFA). The PSOCARE research program aims at the improvement of assistance services for psoriatic patients. It evaluates the factors that help to predict responses to different systemic treatments, their efficacy and safety on a large-scale basis. The program co-ordinates and standardizes different strategies in the management of psoriasis in Italy. The network of various clinical centers involved in this program represents a basis for co-ordinated multicenter clinical studies on the biological drugs in psoriasis. The program is outlined as a cohort study that foresees an active three year-long follow-up of all psoriatic patients treated with biological drugs or other systemic therapy for $8,16,32,52$, 78, 104, and 208 weeks. All patients who received systemic treatment with PUVA, cyclosporin A, acitretin, methotrexate, efalizumab, etanercept, or infliximab for the first time, are eligible to be included in this program. In the period from August 1st 2004 to September 20th 2007, 9,954 subjects were included in this program. Later on, 974 patients were however excluded from the follow-up, due to insufficient information on the date and/or type of therapy prescribed. The remaining 8,980 subjects (67\% males and 33\% females, mean age 49 years) were analyzed, with 6,547 of them (73\%) contributing to the follow-up (the total number of visits was 19,386). The others (2,433 patients, $27 \%)$ could not contribute to the follow-up because they were participating in the study for a period shorter than two months. Furthermore, 1,430 visits $(7.4 \%)$ were excluded from the follow-up due to deviation from the requisites of the program. The patients eligible for the PSOCARE program and subjected to complete analysis $(n=4,287)$ received biological drugs according to the following distribution: etanercept $(n=2,392)$, infliximab $(n=770)$, and efalizumab $(n=1,125)$. The incidence of adverse reactions was similar for all the three biological drugs (around the 1\%-1.5\%). Six cases of death were observed: (a) two cases due to cardiac arrest (one in the group of etanercept and one in the group of efalizumab); (b) one case due to lung embolism (in a patient treated with acitretin); (c) one case due to bronchopneumonia in a patient with etanercept; (d) one case due to an unspecified infection in a patient with efalizumab; and (e) a case of sepsis from Candida crusei in a patient initially treated with etanercept and subsequently with efalizumab. The second PSOCARE report was published in 2007, and has confirmed a slight increase of total infectious episodes in the group of patients treated with biological drugs $(n=81,0.74 \%)$ in comparison to those treated with conventional medicines $(n=36,0.5 \%)$.

\section{Conclusions and perspectives}

There are currently four biological drugs approved for the treatment of plaque-type psoriasis worldwide, namely alefacept, efalizumab, etanercept, and infliximab. Only three of them, namely etanercept, infliximab, and efalizumab, are approved by EMEA for the treatment of psoriasis in the European Union. The British Association of Dermatology has recently published detailed guidelines covering the criteria of choice of biological drugs (Smith et al 2005). According to these guidelines, efalizumab is preferred for patients with a high risk of latent tuberculosis or evidence of demyelinating diseases. Infliximab is recommended when rapid disease control is requirered, and etanercept is to be chosen for the treatment of stable psoriasis. Taking into consideration the lifelong nature of psoriasis, further research is needed to evaluate the long-term disease control by these drugs. In addition, although numerous clinical trials have provided evidence on the safety profile of these drugs, their long-term side effects are still to be evaluated in patients with psoriasis. Finally, there are no comparative data on the clinical efficacy and safety of the different biological drugs used in the management of psoriasis so far.

Here we focused on the biological drugs with immunespecific molecular targets. It is important to note that not all patients with moderate-to-severe psoriasis respond to these therapies. In some cases, the administration of these drugs is even associated to episodes of worsening of the disease. These clinical observations indicate that, beyond the immune-mediated processes targeted by the biologics, other skin-specific mechanisms, undefined so far, may play a relevant role in the induction, maintenance, and/or amplification of this disorder. According with this pathogenetic complexity, some psoriatic patients with low response to biologic monotherapy may display a relevant improvement when treated with biological drugs in combination with conventional systemic therapy (Robinson et al 2007). This experience is however limited and is not authorized for the treatment of psoriasis presently. Conventional systemic drugs may better counteract some pathogenetic component taking place in the psoriatic epidermis, and hence favor the recovery 
of the proliferation/differentiation homeostasis in the skin (Schwartz et al 1992; Heenen et al 1998). The crucial contribution of epidermis-specific processes to the pathogenesis of psoriasis is however vastly ignored, and certainly needs to be investigated to finally clarify the molecular basis of psoriasis at the local level and eventually provide new molecular targets for future, innovative biological drugs.

\section{Disclosure}

The authors report no conflicts of interest in this work.

\section{References}

Baumgarth N, Bevins CL. 2007. Skin deep but complex. Nature, 449:551-3.

Boehncke WH, Brasie RA, Barker J, et al. 2006. Recommendations for the use of etanercept in psoriasis: a European dermatology expert group consensus. J Eur Acad Dermatol Venereol, 20:988-98.

Bongartz T, Sutton AJ, Sweeting MJ, et al. 2006. Anti-TNF antibody therapy in rheumatoid arthritis and the risk of serious infections and malignancies: systematic review and meta-analysis of rare harmful effects in randomized trials. JAMA, 295:2275-85.

Bruch-Gerharz D, Schnorr O, Suschek C, et al. 2003. Arginase 1 overexpression in psoriasis. Limitation of inducible nitric oxide synthase activity as a molecular mechanism for keratinocyte hyperproliferation. Am J Pathol, 162:203-11.

Cassano N, Loconsole F, Galluccio A, et al. 2006. Once-weekly administration of high-dosage etanercept in patients with plaque psoriasis: results of a pilot experience (power study). Int J Immunopathol Pharmacol, 19:823-7.

Chaudhari U, Romano P, Mulcahy LD, et al. 2001 Efficacy and safety of infliximab monotherapy for plaque-type psoriasis: a randomized trial. Lancet, 9271:1842-47.

Dziadzio M, Smith R. 2007. Meta-analysis is no substitute for a comprehensive national registry. Clin Rheumatol, 26:1134-5.

Dubertret L, Sterry W, Bos JD, et al. 2006. Clinical experience acquired with the efalizumab (Raptiva) (CLEAR) trial in patients with moderate-to-severe plaque psoriasis: results from a phase III international randomized, placebo-controlled trial. Br J Dermatol, 155:170-81.

Girolomoni G, Pastore S, Albanesi C, et al. 2002. Targeting tumor necrosis factor- $\alpha$ as a potential therapy in skin inflammatory skin diseases. Curr Opin Investig Drugs, 3:1590-5.

Gisondi P, Gubinelli E, Cocuroccia B, et al. 2004. Targeting tumor necrosis factor- $\alpha$ in the therapy of psoriasis. Curr Drug Targets Inflamm Allergy, 3:175-83.

Gordon KB, Papp KA, Hamilton TK, et al. 2003. Efalizumab for patients with moderate to severe plaque psoriasis: a randomized controlled trial. JAMA, 290:3133-5.

Gordon KB, West DT. 2001. Biologic therapy in psoriasis. In: Wolverton S (ed). Comprehensive dermatologic drug therapy. Philadelphia, PA: WB Saunders Co, pp. 928-42.

Gottlieb AB, Matheson RT, Lowe N, et al. 2003a. A randomized trial of etanercept as monotherapy for psoriasis. Arch Dermatol, 139:1627-32.

Gottlieb AB, Chaudhari U, Mulcahy LD, et al. 2003b. Infliximab monotherapy provides rapid and sustained benefit for plaque-type psoriasis. $J$ Am Acad Dermatol, 48:829-35.

Gottlieb AB, Evans R, Li S, et al. 2004. Infliximab induction therapy for patients with severe plaque-type psoriasis: a randomized double-blind, placebo-controlled trial. J Am Acad Dermatol, 51:534-42.

Gottlieb AB, Hamilton T, Caro I, et al. 2006. Efalizumab Study Group. Long-term continuous efalizumab therapy in patients with moderate to severe chronic plaque psoriasis. J Am Acad Dermatol, 54:5154-63.

Griffiths CEM, Barker JNWN. 2007. Pathogenesis and clinical features of psoriasis. Lancet, 370:263-71.
Hankin CS, Feldman SR, Szczotka A, et al. 2005. A cost comparison of treatments for moderate to severe psoriasis. Drug Ben Trends, 17:200-14.

Heenen M, Laporte M, Noel JC, et al. 1998. Methotrexate induces apoptotic cell death in human keratinocytes. Arch Dermatol Res, 290:240-5.

Hochberg MC, Lebwohl MG, Plevy SE, et al. 2005. The benefit/risk profile of TNF-blocking agents: findings of a consensus panel. Semin Arthritis Rheum, 34:819-36.

Hogg N, Laschinger M, Giles K, et al. 2003. T cell integrins: more than just sticking points. $J$ Cell Sci, 116:4695-705.

Krueger G, Elewski B, Papp K, et al. 2006. Patients with psoriasis respond to continuous open-label Etanercept treatment after initial incomplete response in randomized, placebo controlled trial. J Am Acad Dermatol, 54:S112-9.

Lande R, Gregorio J, Facchinetti V, et al. 2007. Plasmacytoid dendritic cells sense self-DNA coupled with antimicrobial peptide. Nature, 449:564-9.

Langley RG, Carey WP, Rafal ES, et al. 2005. Incidence of infection during efalizumab therapy for psoriasis: analysis of the clinical trial experience. Clin Ther, 27:1317-28.

Lebwohl M, Tyring SK, Hamilton TK, et al.; Efalizumab Study Group. 2003. A novel targeted T-cell modulator, efalizumab, for plaque psoriasis. N Engl J Med, 349:2004-13.

Leonardi CL, Powers JL, Matheson RT, et al. 2003. Etanercept as monotherapy in patients with psoriasis. $N$ Engl J Med, 349:2014-22.

Lewis TG, Tuchinda HW, Lim HKW. 2005. Life-threatening pustolar and erythrodermic psoriasis responding to infliximab. J Drugs Dermatol, 5:546-8.

Liu Y, Krueger JG, Bowcock AM. 2007. Psoriasis: genetic associations and immune system changes. Genes Immun, 8:1-12.

Locksley RM, Killeen N, Lenardo MJ. 2001. The TNF and TNF receptor superfamilies: integrating mammalian biology. Cell, 104:487-501.

Magliocco MA, Gottlieb AB. 2004. Etanercept therapy for patients with psoriatic arthritis and concurrent hepatitis $\mathrm{C}$ virus infection: report of three cases. J Am Acad Dermatol, 51:580-4.

Menter A, Gordon K, Carey W, et al. 2005. Efficacy and safety observed during 24 weeks of efalizumab therapy in patients with moderate to severe plaque psoriasis. Arch Dermatol, 141:31-8.

Menter A, Feldman SR, Weinstein GD, et al. 2007. A randomized comparison of continuous vs. intermittent infliximab mainenance regimens over 1 year in the treatment of moderate-to-severe plaque psoriasis. J Am Acad Dermatol, 56:31-44.

Menter A, Griffiths CE. 2007. Current and future management of psoriasis. Lancet, 370:272-84.

Mueller W, Herrmann B. 1979. Cyclosporin A for psoriasis. N Engl J Med, 301:555.

Nelson AA, Pearce DJ, Fleischer AB, et al. 2008. Cost-effectiveness of biological treatments for psoriasis based on subjective and objective efficacy measures assessed over a 12 -week treatment period. $J \mathrm{Am}$ Acad Dermatol, 58:125-5.

Ong PY, Ohtake T, Brandt C, et al. 2002. Endogenous antimicrobial peptides and skin infections in atopic dermatitis. N Engl J Med, 347:1151-60.

Papp KA, Tyring S, Lahfa M, et al. 2005. A global phase III randomized controlled trial of etanercept in psoriasis: safety, efficacy, and effect of dose reduction. Br J Dermatol, 152:1304-12.

Papp KA. 2006. The long-term efficacy and safety of new biological therapies for psoriasis. Arch Dermatol Res, 298:7-15.

Pasparakis M, Curtois G, Hafner M, et al. 2002. TNF-mediated inflammatory skin disease in mice with epidermis-specific deletion of IKK2. Nature, 417:861-6.

Pastore S, Mascia F, Mariani V, et al. 2006. Keratinocytes in skin inflammation. Expert Rev Dermatol, 1:279-91.

Pincelli C, Henninger E, Casset-Semanaz F. 2006. The incidence of arthropathy adverse events in efalizumab-treated patients is low and similar to placebo and does not increase with long-term treatment: pooled analysis of data from Phase III clinical trials of efalizumab. Arch Dermatol Res, 298:329-38. 
Reich K, Nestle FO, Papp K, et al. 2005. EXPRESS study investigators. Infliximab induction and maintenance therapy for moderate-to-severe psoriasis: a phase III, multicentre, double-blind trial. Lancet, 366:1367-74.

Robinson MR, Korman BD, Korman NJ. 2007. Combination immunosuppressive therapies. Arch Dermatol, 143:1053-7.

Rokhsar C, Rabhan N, Cohen SR. 2006. Etanercept monotherapy for a patient with psoriasis, psoriatic arthritis, and concomitant hepatitis $\mathrm{C}$ infection. J Am Acad Dermatol, 54:361-2.

Schon MP, Ludwig RJ. 2005. Lymphocyte trafficking to inflamed skin. Molecular mechanisms and implications for therapeutic target molecules. Expert Opin Ther Targets, 9:225-43.

Schwartz PM, Barnett SK, Attillasoy ES, et al. 1992. Methotrexate induces differentiation of human keratinocytes. Proc Natl Acad Sci U S A 89:594-8.

Shikiar R, Willian MK, Okun MM, et al. 2006. The validity and responsiveness of three quality of life measures in the assessment of psoriasis patients: results of a phase II study. Health Qual Life Outcomes, 4:71.

Skomsvoll JF, Wallenius M, Koksvik HS, el al. 2007. Drug Insight: antitumor necrosis factor therapy for inflammatory arthropathies during reproduction, pregnancy and lactation. Nat Clin Pract Rheumatol, $3: 156-64$.
Smith CH, Anstey AV, Barker JNWN, et al. 2005. British Association of Dermatology Guidelines for the use of biological interventions in psoriasis 2005. Br J Dermatol, 153:486-97.

Sterry W, Stingl G, Langley RG, et al.; CLEAR Multinational Study Group. 2006. Clinical Experience Acquired with Raptiva (CLEAR) trial in patients with moderate-to-severe plaque psoriasis: results from extended treatment in an international, Phase III, placebo-controlled trial. J Dtsch Dermatol Ges, 4:947-56.

Travers JB. 2000. Novel immunomodulators for topical skin disease therapy. Expert Opin Invest Drugs, 9:529-42.

Valdimarsson H. 2007. The genetic basis of psoriasis. Clin Dermatol, 25:563-7.

Wahie S, Alexandruff A, Reynolds NJ, et al. 2006. Psoriasis occurring after myeloablative therapy and autologous stem cell transplantation. Br J Dermatol, 154:194-5.

Weinberg JM. 2006. A review of the safety of the tumor necrosis inhibitors infliximab, etanercept, and adalimumab. In: Weinberg JM, Buchholz R, (Eds). TNF-alpha inhibitors. Basel: Birkhäuser, pp. 115-27.

Zeichner JA, Lebwohl M. 2007. Potential complications associated with the use of biologic agents for psoriasis. Dermatol Clin, 25:207-13. 
